\title{
CROSS-CALIBRATION OF IMAGING SENSORS USING MODEL- BASED, SI-TRACEABLE PREDICTIONS OF AT-SENSOR RADIANCE
}

\author{
Kurtis Thome, Joel McCorkel
}

NASA, Goddard Space Flight Center

Many inter-consistency efforts force empirical agreement between sensors viewing a source nearly coincident in time and geometry that ensures consistency between sensors rather than obtain an SItraceable calibration with documented error budgets. The method described here provides interconsistency via absolute radiometric calibration with defensible error budget avoiding systematic errors through prediction of at-sensor radiance for a site viewed by multiple sensors but not necessarily viewed at coincident times. The method predicts spectral radiance over a given surface site for arbitrary view and illumination angles and for any date dominated by clear-sky conditions. The foundation is a modelbased, SI-traceable prediction of at-sensor radiance over selected sites based on physical understanding of the surface and atmosphere. The calibration of the ground site will include spatial, spectral, and sunview geometric effects based on satellite and ground-based data. The result is an interconsistency of hyperspectral and multispectral sensors spanning spatial resolutions from meters to kilometers all relative to the surface site rather than a single sensor. The source-centric philosophy of calibrating the site inherently accounts for footprint size mismatch, spectral band mismatch, and temporal and spatial sampling effects. The method for characterizing the test site allows its use for SI-traceable calibration of any sensor that can view the calibrated test site. Interconsistency is obtained through the traceability and error budget rather than coincident views. Such an approach to inter-consistency provides better understanding of biases between sensors as well producing more accurate results with documented SItraceability that reduces the need for overlapping data sets. 
There exist numerous methods for on-orbit characterization ranging from methods that include measurements of surface and atmospheric properties at the time of a sensor overpass to invariant scene approaches relying on knowledge of temporal characteristics of the site. This presentation examines typical cross-calibration methods and expected uncertainties of the methods. Proposed methods to mitigate the largest error sources in future systems are presented. A case study example using Landsat data is provided that act as the basis for a set of recommendations to ensure future SI-traceable cross calibration using future missions such as CLARREO and TRUTHS. 